\title{
HOMOTOPY EQUIVALENCES IN EQUIVARIANT TOPOLOGY
}

\author{
MARTIN FUCHS
}

\begin{abstract}
Homomorphisms up to homotopy (higher homotopies that is) are generalized for the equivariant category. Homotopy equivalences have an inverse in this new category.
\end{abstract}

Introduction. In equivariant topology the notion of a homotopy equivalence presents a problem. Strictly within the equivariant category, homotopy equivalence seems to be too limited a concept, ${ }^{1}$ e.g. the unit interval $I$ (acting on itself as an $H$-space) is not of the same equivariant homotopy type as $\{1\}$ as a $\{1\}$-space.

Some of the rather general tools used in homotopy theory of topological groups and $H$-spaces (e.g. studying classifying spaces) can also be used in equivariant homotopy theory. In this paper we use $G_{\infty}$-maps between $G$-spaces (as defined in 1.4, and similar to $H_{\infty}$-maps between $H$-spaces) to study a new notion of homotopy equivalence. Roughly speaking, if $X$ and $\bar{X}$ are $G$-spaces and if $f: X \rightarrow \bar{X}$ is a $G$-equivariant map and also an ordinary homotopy equivalence, then there exists a sequence of maps $g_{n}: \bar{X} \times(I \times G)^{n} \rightarrow X$ forming a $G_{\infty}$-map such that $f$ and the maps $\left\{g_{n}\right\}$ form a pair of $G_{\infty}$-homotopy equivalences.

The complete theorem is stated in $\$ 2$. The proof includes the proof of the corresponding theorem on $H$-spaces as stated in [2, Theorem 4.1] or in [1] as Proposition 1.17. We hope to convince the reader that this proof is not as messy as it is described by the authors of [1] on p. 13.

\section{Definitions.}

Definition 1.1. An $H$-space $G$ is a topological space with a continuous multiplication $\mu$. We assume that $\mu$ is strictly associative. No unit element is needed.

Definition 1.2. A topological space $X$ is called a $G$-space, if $G$ acts on $X$ in a continuous and in an associative manner.

Multiplication and actions will be denoted by the usual juxtaposition.

Definition 1.3. An $H_{\infty}$-map $\underline{h}$ from $G$ to $\bar{G}$ of length $r^{2}$ is a sequence of continuous maps $h_{n}: G \times\left(I_{r} \times G\right)^{n} \rightarrow \bar{G}$ such that

Presented to the Society, August 22, 1975; received by the editors August 13, 1974 and, in revised form, November 4, 1975.

AMS (MOS) subject classifications (1970). Primary 55D45, 55D10; Secondary 57E99.

${ }^{1}$ C. N. Lee, A. Wasserman (see [5]), and T. Petrie have relatively simple examples of pairs of manifolds $X$ and $Y$ with $S^{1}$-actions and an $S^{1}$-map $f: X \rightarrow Y$ such that $f$ is an ordinary homotopy equivalence. But there is no equivariant map $g: Y \rightarrow X$ which is a homotopy equivalence.

2 We will mention the length of maps only if it is essential to the context. 


$$
\begin{aligned}
h_{n}\left(g_{0}, t_{1}, \ldots, t_{n}, g_{n}\right) & \begin{array}{ll}
h_{n-1}\left(g_{0}, t_{1}, \ldots, g_{i-1} g_{i}, \ldots, t_{n}, g_{n}\right), & t_{i}=0, \\
h_{i-1}\left(g_{0}, t_{1}, \ldots, g_{i-1}\right) h_{n-i}\left(g_{i}, \ldots, g_{n}\right), & t_{i}=r,
\end{array} \quad n \geqslant 0,
\end{aligned}
$$

where $g_{0}, \ldots, g_{n} \in G$ and $t_{1}, \ldots, t_{n} \in I_{r}=[0, r] \subset \mathbf{R}$. If $r=0$, the map $h_{0}$ is an ordinary homomorphism.

Definition 1.4. Let $X$ be a $G$-space, $\bar{X}$ be a $\bar{G}$-space and let $\underline{h}$ be an $H_{\infty}$-map from $G$ to $\bar{G}$ of length $r$. A $G_{\infty}$-map $\underline{f}$ from $X$ to $\bar{X}$ of length $r$ associated to $\underline{h}$ is a sequence of maps $f_{n}: X \times\left(I_{r} \times \bar{G}\right)^{n} \rightarrow \bar{X}$ such that

$$
\begin{aligned}
& f_{n}\left(x, t_{1}, g_{1}, \ldots, t_{n}, g_{n}\right) \\
& \quad=\left\{\begin{array}{ll}
f_{n-1}\left(x, t_{1}, \ldots, g_{i-1} g_{i}, \ldots, t_{n}, g_{n}\right), & t_{i}=0, \\
f_{i-1}\left(x, t_{1}, g_{1}, \ldots, g_{i-1}\right) h_{n-i}\left(g_{i}, \ldots, t_{n}, g_{n}\right), & t_{i}=r,
\end{array} n \geqslant 0 .\right.
\end{aligned}
$$

If $t_{1}=0$ we want of course

$$
f_{n}\left(x, 0, g_{1}, t_{2}, \ldots, g_{n}\right)=f_{n-1}\left(x g_{1}, t_{2}, \ldots, g_{n}\right) .
$$

So $G_{\infty}$-maps formally differ from $H_{\infty}$-maps only in the first coordinate.

Composition of $G_{\infty}$-maps is defined the same way as for $H_{\infty}$-maps. If $n=1$

$$
(\bar{f} \circ f)_{1}\left(x, t_{1}, g_{1}\right)= \begin{cases}\bar{f}_{0}\left(f_{1}\left(x, t_{1}, g_{1}\right)\right), & 0 \leqslant t_{1} \leqslant r, \\ \bar{f}_{1}\left(f_{0}(x), t_{1}-r, h_{0}(y)\right), & r \leqslant t_{1} \leqslant r+s,\end{cases}
$$

which is the standard composition of homotopies. If $n>1$ we have to form the composite of $2^{n}$ maps, each of which is defined on one of the $2^{n}$ rectangular boxes obtained from $I_{r+s}^{n}$ be partitioning it with the hyperplanes $t_{i}=r$.

Definition 1.5. Let $X_{1}$ be a $G_{1}$-space, $X_{2}$ a $G_{2}$-space, and $X_{3}$ a $G_{3}$-space. Let $\underline{h}$ be an $H_{\infty}$-map of length $r$ from $G_{1}$ and $G_{2}$ and $\underline{\bar{h}}$ an $H_{\infty}$-map of length $s$ from $G_{2}$ to $G_{3}$. Also let $\underline{f}$ be a $G_{\infty}$-map of length $r$ from $X_{1}$ to $X_{2}$ associated to $\underline{h}$, and let $\bar{f}$ be a $G_{\infty}$-map of length $s$ from $X_{2}$ to $X_{3}$ associated to $\underline{\bar{h}}$. We define $(\bar{f} f)_{n}: X_{1} \times\left(I_{r+s} \times G_{1}\right)^{n} \rightarrow X_{3}$ by

$$
\begin{aligned}
& (\bar{f} \circ f)_{n}\left(x, t_{1}, g_{1}, \ldots, t_{n}, g_{n}\right) \\
& =\bar{f}_{j}\left(f_{i_{1}}\left(x, t_{1}, g_{1}, \ldots, t_{i_{1}}, g_{i_{1}}\right), t_{i_{1}+1}-r, h_{i_{2}-i_{1}-1}(\cdots), \ldots,\right. \\
& \left.t_{i_{j}+1}-r, h_{n-i_{j}-1}\left(g_{i_{j}+1}, \ldots, t_{n}, g_{n}\right)\right) .
\end{aligned}
$$

Here $x \in X_{1}, g_{i} \in G_{1}$, and for every sequence of natural numbers $0 \leqslant i_{1}$ $<\cdots<i_{j}<n(j=1, \ldots, n)$ we have $t_{i_{1}+1}, \ldots, t_{i_{j}+1} \in[r, r+s]$, while $t_{i}$ $\in[0, r]$ whenever $i-1 \neq i_{1}, \ldots, i_{j}$.

Composition formulas for $H_{\infty}$-maps can also be found in [2], [3] and [4].

We leave it to the reader to verify that the maps $\left\{(\bar{f} f)_{n}\right\}$ form a $G_{\infty}$-maps associated to $\left\{(\bar{h} h)_{n}\right\}$. The composition of $G_{\infty}$-maps is associative and together with the $G$-spaces they form a category.

Definition 1.6. Let $\underline{h}^{0}$ and $\underline{h}^{1}$ be $H_{\infty}$-maps from $G$ to $\bar{G}$, and let $\underline{f}^{0}$ and $\underline{f}^{1}$ be $G_{\infty}$-maps from $X$ to $\bar{X}$ associated to $\underline{h}^{0}$ and $\underline{h}^{1}$ respectively. If $\underline{h}^{t}(0 \leqslant t$ $\leqslant 1)$ is a family of $H_{\infty}$-maps constituting an $H_{\infty}$-homotopy between 
$\underline{h}^{0}$ and $\underline{h}^{1}$, then a family $\underline{f}^{t}(0 \leqslant t \leqslant 1)$ of $G_{\infty}$-maps from $X$ to $\bar{X}$ associated with $\underline{h}^{t}$ is called a $G_{\infty}$-homotopy between $\underline{f}^{0}$ and $\underline{f}^{l}$.

Definition 1.7. A $G_{\infty}$-map $\underline{f}$ from $X$ to $\bar{X}$ associated with the $H_{\infty}$-map $\underline{h}$ from $G$ to $\bar{G}$ is called a $G_{\infty}$-homotopy equivalence if there exists an $H_{\infty}$-map $\underline{\bar{h}}$ from $\bar{G}$ to $G$ and a $G_{\infty}$-map $\bar{f}$ from $\bar{X}$ to $X$ such that the respective compositions are $G_{\infty}$-homotopic (and $H_{\infty}$-homotopic respectively) to $\mathrm{id}_{X}$ and $\mathrm{id}_{\bar{X}}$ associated to $\mathrm{id}_{G}$ and $\mathrm{id}_{\bar{G}}$ respectively. The $G_{\infty}$-map $\underline{\underline{f}}$ associated with $\underline{\bar{h}}$ is called a homotopy inverse of $\underline{f}$ associated with $\underline{h}$.

\section{Theorems and proofs.}

THEOREM. Let $X$ be a $G$-space and $\bar{X}$ be a $\bar{G}$-space. If $\underline{h}$ is an $H_{\infty}$-map from $G$ to $\bar{G}$ such that $h_{0}$ is an ordinary homotopy equivalence, and if $f$ is a $G_{\infty}$-map from $X$ to $\bar{X}$ associated with $\underline{h}$ such that $f_{0}$ is an ordinary homotopy equivalence, then $\underline{f}$ is a $G_{\infty}$-homotopy equivalence.

REMARK. We construct both an inverse $\underline{\bar{h}}$ to $\underline{h}$ and an inverse $\bar{f}$ to $f$ associated with $\underline{\underline{h}}$. However, the construction of $\underline{f}$ works for any inverse of $\underline{\underline{h}}$, and also for every homotopy inverse of $f_{0}$.

Two special cases are of importance.

Corollary. If $X$ and $\bar{X}$ are $G$-spaces and $f: X \rightarrow \bar{X}$ is a G-equivariant map as well as an ordinary homotopy equivalence, then $f$ is a $G_{\infty}$-homotopy equivalence associated to $1_{G}$.

CoRollaRy. If $H$ and $\bar{H}$ are $H$-spaces and $h: H \rightarrow \bar{H}$ is a strict homomorphism (or an $H_{\infty}$-map) as well as an ordinary homotopy equivalence, then $h$ is an $H_{\infty}$-homotopy equivalence (see [2, Theorem 4.1] and [1]).

The second corollary is obtained by choosing $X=H$ and $\bar{X}=\bar{H}$.

Proof. We will construct an $H_{\infty}$-map $\underline{\underline{h}}: \bar{G} \rightarrow G$ which is an $H_{\infty}$-homotopy inverse to $\underline{h}$, and a $G_{\infty}-\operatorname{map} \bar{f}: \bar{X} \rightarrow X$ associated to $\underline{\underline{h}}$ which is a $G_{\infty}$-homotopy inverse to $f$. The construction is by induction.

$n=0:$ Let $\bar{f}_{0}: \bar{X} \rightarrow X$ be an ordinary homotopy inverse to $f_{0}$ and $k_{0}: X \times I$ $\rightarrow X$ be such that

$$
k_{0}(x, t)=\left\{\begin{array}{ll}
x & \text { for } t=0, \\
\bar{f}_{0} f_{0}(x) & \text { for } t=1,
\end{array} \quad x \in X .\right.
$$

Then, since $f_{0}$ and $\bar{f}_{0}$ induce isomorphisms on the relevant homotopy classes of maps (compare e.g. [2, p. 205]) we can choose $\bar{k}_{0}: \bar{X} \times I \rightarrow \bar{X}$ such that

$$
\bar{k}_{0}(\bar{x}, t)= \begin{cases}\bar{x} & \text { for } t=0 \\ f_{0} \bar{f}_{0}(\bar{x}) & \text { for } t=1\end{cases}
$$

in such a manner that there exists a map $u_{0}: X \times I^{2} \rightarrow \bar{X}$ with

$$
\begin{aligned}
& u_{0}\left(x, 0, s_{2}\right)=f_{0} k_{0}\left(x, s_{2}\right), \\
& u_{0}\left(x, 1, s_{2}\right)=\bar{k}_{0}\left(f_{0}(x), s_{2}\right),
\end{aligned}
$$

and 


$$
\begin{aligned}
& u_{0}\left(x, s_{1}, 1\right)=f_{0} \bar{f}_{0} f_{0}(x) \\
& u_{0}\left(x, s_{1}, 0\right)=f_{0}(x)
\end{aligned}
$$

We will call $\bar{f}_{0}$ together with $\left(k_{0}, \bar{k}_{0}\right)$ and $u_{0}$ a compatible homotopy inverse to $f_{0}$.

Similarly we choose a compatible homotopy inverse $\bar{h}_{0}$ together with homotopies $\left(l_{0}, \bar{l}_{0}\right)$ and $v_{0}$ for the map $h_{0}$ (compare [2, p. 205]). We notice that this second choice is independent of the choice of $\bar{f}_{0}$.

INDUCTION HYPOTHESIS. Assume we already constructed $\left(\bar{f}_{0}, \ldots, \bar{f}_{n-1}\right)$ as the first $n$ functions of a $G_{\infty}$-homotopy inverse for $f$ associated with $\left(\bar{h}_{0}, \ldots, \bar{h}_{n-1}\right)$, the $n$ functions of an $H_{\infty}$-homotopy inverse of $\underline{h}$. Furthermore assume that we constructed these functions as compatible inverses to $\left(f_{0}, \ldots, f_{n-1}\right)$ and $\left(h_{0}, \ldots, h_{n-1}\right)$. For convenience sake we assume that all maps of $\underline{f}$ and $\underline{h}$ have length one. Also $\bar{f}_{0}, \ldots, \bar{f}_{n-1}$ and $\bar{h}_{0}, \ldots, \bar{h}_{n-1}$ are assumed to have length one. The homotopies $k_{0}, \ldots, k_{n-1}, \bar{k}_{0}, \ldots, \bar{k}_{n-1}, l_{0}$, $\ldots, l_{n-1}$ and $\bar{l}_{0}, \ldots, \bar{l}_{n-1}$ are to be of length two. The maps $u_{0}, \ldots$, $u_{n-1}$ and $v_{0}, \ldots, v_{n-1}$ are to have length three. These last maps shall satisfy the obvious boundary conditions as described in 1.3 and 1.4, in addition to the compatibility conditions.

Now we will construct $u_{n}: X \times\left(I_{3} \times G\right)^{n} \times I^{2} \rightarrow \bar{X}$. (The maps $\bar{f}_{n}, k_{n}$ and $\bar{k}_{n}$ will be defined in the process of this construction.) For our construction by induction $u_{n}$ shall have the following properties.

$$
u_{n} \mid X \times\left(I_{3} \times G\right)^{n} \times I \times\{0\}=\left(\bar{e}_{1} \circ f \circ e_{1}\right)_{n} \times \mathrm{id}_{I} .
$$

Here $e_{1}$ and $\bar{e}_{1}$ stands for the identity morphism of $X$ and $\bar{X}$ respectively with length 1 . We observe that this part of $u_{n}$ is already known.

$$
u_{n} \mid X \times\left(I_{3} \times G\right)^{n} \times I \times\{1\}=(f \circ \bar{f} \circ f)_{n} \times \mathrm{id}_{I} .
$$

The composition $(f \circ \bar{f} \circ f)_{n}$ contains the map $\bar{f}_{n}$ only if $1 \leqslant t_{i} \leqslant 2$ (for $i=1, \ldots, n)$. For these values of $t_{i}$ we have

$$
(f \circ \bar{f} \circ f)_{n}\left(x, t_{1}, g_{1}, \ldots, g_{n}\right)=f_{0} \bar{f}_{n}\left(f_{0}(x), t_{1}, h_{0}\left(g_{1}\right), \ldots, t_{n}, h_{0}\left(g_{n}\right)\right) .
$$

The rest of $(f \circ \bar{f} \circ f)_{n}$ uses $\bar{f}_{k}$ with $1 \leqslant k<n$. Because of this and the boundary properties of $\bar{f}_{n}$ we know this part of $u_{n}$ except when $1<t_{i}<2, i$ $=1, \ldots, n$.

$$
u_{n} \mid X \times\left(I_{3} \times G\right)^{n} \times\{0\} \times I=\left(\bar{e}_{1-s_{2}} \circ f \circ k_{1+s_{2}}\right)_{n} .
$$

$\bar{e}_{1-s_{2}}$ stands for the identity morphism of $\bar{X}$ with length $1-s_{2}$, and $k_{1+s_{2}}$ stands for the homotopy $k$ with length $1+s_{2}$, where $s_{2}$ is the second coordinate of $I \times I$. From the composition rule for $G_{\infty}$-maps we see that this restriction of $u_{n}$ is known except for $1<t_{i}<2$ when $s_{2}=1$, and $2-s_{2}<t_{i}$ $<3$ when $0<s_{2}<1(i=1, \ldots, n)$, i.e. when

$$
\begin{aligned}
& u_{n}\left(x, t_{1}, g_{1}, \ldots, t_{n}, g_{n}, 0, s_{2}\right) \\
& \quad= \begin{cases}f_{0} \bar{f}_{n}\left(f_{0}(x), t_{1}, h_{0}\left(g_{1}\right), \ldots, t_{n}, h_{n}\left(g_{n}\right)\right), & s_{2}=1, \\
f_{0} k_{1+s_{2}, n}\left(x, t_{1}, \ldots, t_{n}, g_{n}, s_{2}\right), & 0<s_{2}<1 .\end{cases}
\end{aligned}
$$




$$
u_{n} \mid X \times\left(I_{3} \times G\right)^{n} \times\{1\} \times I=\left(\bar{k}_{1+s_{2}} \circ f \circ e_{1-s_{2}}\right)_{n}
$$

which is known except for $1<t_{i}<2$ when $s_{2}=1$, and $0<t_{i}<1+s_{2}$ when $0<s_{2}<1$, i.e. when

$$
\begin{aligned}
& u_{n}\left(x, t_{1}, g_{1}, \ldots, t_{n}, g_{n}, 1, s_{2}\right) \\
& \quad= \begin{cases}f_{0} \bar{f}_{n}\left(f_{0}(x), t_{1}, h_{0}\left(g_{1}\right), \ldots, t_{n}, h_{0}\left(g_{n}\right)\right), & s_{2}=1, \\
\bar{k}_{1+s_{2}, n}\left(f_{0}(x), t_{1}, h_{0}\left(g_{1}\right), \ldots, t_{n}, h_{0}\left(g_{n}\right)\right), & 0<s_{2}<1 .\end{cases}
\end{aligned}
$$

(5) $u_{n} \mid X \times \partial\left(I_{3} \times G\right)^{n} \times I^{2}$ is defined by $u_{0}, \ldots, u_{n-1}$ and $v_{0}, \ldots, v_{n-1}$ according to the properties of $G_{\infty}$ - and $H_{\infty}$-maps as described in 1.3 and 1.4. Hence this part of $u_{n}$ is known.

To find all of $u_{n}$, we are going to use the following consequence of the homotopy extension property:

Lemma. If $A \subset X$ has the homotopy extension property and if $r: Y_{1} 1 \leftrightarrows Y_{2}: s$ are homotopy equivalences, then if $f_{1}: A \rightarrow Y_{1}$ is a map such that $r \circ f_{1}$ has an extension $g_{2}: X \rightarrow Y_{2}$, we know that $f_{1}$ has an extension $g_{1}: X \rightarrow Y_{1}$ by the homotopy extension property.

We observe that the extension problem resulting from (3) is homeomorphic to extending a map, which is known on all of $Y \times \partial I^{n+1}$ with the exception of one face of $Y \times I^{n+1}$, to all of $Y \times I^{n+1}$. We extend, and use the lemma to obtain $K_{1+s_{2}}: X \times\left(I_{1+s_{2}} \times G\right)^{n} \times\{0\} \times I \rightarrow X$. For $s_{2}=1$ we see that $K_{1+s_{2}}$ provides an extension of $\bar{f}_{n} \circ f_{0}$ from $X \times \partial([1,2] \times G)^{n}$ to $X \times([1,2] \times G)^{n^{2}}$. To obtain the extension of $\partial \bar{f}_{n}: \bar{X} \times \partial([1,2] \times \bar{G})^{n} \rightarrow X$ we use the following

Lemma. Given a homotopy equivalence $a: A \rightarrow \bar{A}$ a map $\partial c: \bar{A} \times \partial I^{n} \rightarrow \bar{B}$, and a map $w: A \times I^{n} \rightarrow \bar{B}$ that extends $\partial c(a \times 1)$, then $\partial c$ extends to a map $c: \bar{A} \times I^{n} \rightarrow \bar{B}$ such that $c(a \times 1)$ is homotopic to $w$ keeping the boundary fixed.

Since on $X \times \partial([1,2] \times G)^{n} \times\{1\} \times\{1\}$ we have

$$
K_{1+s_{2}}\left(x, t_{1}, g_{1}, \ldots, t_{n}, g_{n}, 1,1\right)=\bar{f}_{n}\left(f_{0}(x), t_{1}, h_{0}\left(g_{1}\right), \ldots, t_{n}, h_{0}\left(g_{n}\right)\right),
$$

we obtain that $K_{1+s_{2}} \mid X \times([1,2] \times G)^{n} \times\{1\} \times\{1\}$ is homotopic to $\bar{f}_{n} \circ f_{0}$ $\times\left(1 \times h_{0}\right)^{n}$ relative to the boundary $X \times \partial([1,2] \times G)^{n} \times\{1\} \times\{1\}$. We combine $K_{1+s_{2}}$ with such a homotopy to obtain $k_{1+s_{2}}$, with $k_{1+s_{2}} \mid X \times([1,2] \times G)^{n}$ $\times\{1\} \times\{1\}=\bar{f}_{n} \circ f_{0} \times\left(1 \times h_{0}\right)^{n}$.

We now know $u_{n}$ except on $X \times\left(I_{3} \times G\right)^{n} \times I^{2}$ and the part of $X$ $\times\left(I_{3} \times G\right)^{n} \times\{1\} \times I$ with $0<t_{i}<1+s_{2}$ and $0<s_{2}<1$. This extension problem is homeomorphic to the problem of extending a map which is known on all of $Y \times \partial I^{n+2}$ with the exception of one face to all of $Y \times I^{n+2}$. Let $U_{n}$ be such an extension. We have to use the second lemma once more to obtain $\bar{k}_{1+s_{2}}$. Again $\bar{k}_{1+s_{2}, n}\left(f_{0} \times 1_{I} \times h_{0} \times \cdots \times h_{0}\right)$ is homotopic to the right restriction of $U_{n}$ leaving the boundary fixed. We alter $U_{n}$ by this homotopy and obtain $u_{n}$.

The construction of $v_{n}, \bar{h}_{n}, l_{n}$, and $\bar{l}_{n}$ is completely analogous and is left to the reader. 


\section{REFERENCES}

1. J. M. Boardman and R. M. Vogt, Homotopy invariant algebraic structures on topological spaces, Lecture Notes in Math., vol. 347, Springer-Verlag, Berlin and New York, 1973.

2. M. Fuchs, Verallgemeinerte Homotopie-Homomorphismen und klassifizierende Räume, Math. Ann. 16 (1965), 197-230. MR 33 \#3295.

3. A. Clark, Homotopy commutativity and the Moore spectral sequence, Pacific J. Math. 15 (1965), 65-74. MR 31 \#1679.

4. B. Drachman, A generalization of the Steenrod classification theorem to H-spaces, Trans. Amer. Math. Soc. 153 (1971), 53-88. MR 44 \#5961.

5. C. N. Lee and A. G. Wasserman, On the groups $J O(G)^{*}$, University of Michigan (to appear).

Department of Mathematics, Michigan State University, East Lansing, Michigan 48824 